\title{
A CHECKLIST OF THE TASMANIAN TORTRICID MOTHS (LEPIDOPTERA: TORTRICIDAE) AND THEIR HOST-PLANT RELATIONSHIPS
}

\author{
by P.B. McQuillan
}

\begin{abstract}
MCQUILlAN, P.B., 1992 (31:x): A checklist of the Tasmanian tortricid moths (Lepidoptera: Tortricidae) and their host-plant relationships. Pap. Proc. R. Soc. Tasm. 126: 77-89. https://doi.org/10.26749/rstpp.126.77

Plant Protection Branch, Department of Primary Industry, GPO Box 192B, Hobart, Tasmania, Australia 700 I.
\end{abstract}

\begin{abstract}
An annotated checklist of 165 described species of Tasmanian Tortricidae is presented. There is a single endemic genus, the monobasic Symphygas, and the genera Coeloptera, Isotenes, Neohermenias and Tracholena are recorded for the first time. At least $6 \mathbf{s p e c i e s ~ ( i . e . ~ a b o u t ~}$ one-third of the fauna) are endemic to Tasmania. The majority of species belong to the tortricine tribesArchipini ( 66 species) and Epitymbiini (49species) while only 26 nativespecies of Olethrentinae are recorded. Two species are introduced from Europe. The plant families Asteraceae and Myrtaceae are pre-eminent as foodplants. New foodplants are recorded for a number of species and the degree of monophagy is shown to be high. Foodplants are now known for abour one-third of the fauna.
\end{abstract}

Key Words: Lepidoptera, Tortricidae, checklist, Tasmania, foodplants.

\section{INTRODUCTION}

The Tortricidae are a large family of small moths (c. 5000 species worldwide) represented in most vegetated parts of the world. About 600 species of Tortricidae are named from Australia at present, but the total fauna may be double this number (Common 1980). The larvae, familiar as leaf-rolling caterpillars, characteristically form small shelters from leaves of their foodplant bound with silk, or bore into plant tissue. Collectively, a very wide range of plants are used as hosts but most foodplants are perennial shrubs or trees.

Several native Australian species, including the light-brown apple moth, the orange fruitborer and the macadamia nutborer, have adopted new foodplants and become significant pests of agriculture, especially horticultural crops, causing economic losses domestically; they are therefore subject to quarantine barriers in export markets. Some others, such as the codling moth and the oriental fruit moth, are adventive with European man.

Despite the real and potential economic importance of the Tortricidae, there has been only a limited amount of systematic and biological work done on the Tasmanian fauna. Although Tasmania was a source of specimens for the early descriptive works of Walker (1863) and Meyrick (1881, 1910), the first and only available catalogue of species is that of Turner (1928) with its later supplement (Turner 1939) in which many species were assigned to European genera now known to be inappropriate. Since that time, a number of new species and occurrences have been discovered, and the generic and tribal classification of the family in Australia is much better understood. In recent years, the host-plant relationships of various species have been the subject of active research and have illuminated aspects as diverse as phylogeny and pest potential.

The purpose of this study was (i) to prepare a current annotated checklist of the species of Tortricidae known to be present in Tasmania, and (ii) to determine the foodplant relationships for as many species as possible. In view of the increasing diversity of Tasmanian horticultural products, such a study is timely and a necessary prerequisite for assessing quarantine risks.

\section{MATERIALS AND METHODS}

A species checklist was prepared from field collections, museum specimens and from literature sources. The arrangement of genera and species within genera is alphabetical, pending better resolution of the phylogeny of the taxa; original generic assignments are in brackets and synonyms are indented. For many taxa, known relationships between them have not been formalised as genera and, instead, are referred to nominal species groups. These informal groupings are adopted with permission from the unpublished work of Dr I.F.B. Common, formerly of the CSIRO Division of Entomology, although some have been previously used in Powell \& Common's (1985) study of oviposition behaviour.

Larvae were collected directly from the field and reared to adulthood on samples of their foodplants. Voucher specimens of moths are preserved at the New Town Research Laboratories of the Tasmanian Department of Primary Industry. Foodplant citations follow Buchanan et al. (1989). For categorising the degree of host specificity, the criteria of Powell (1980) were applied. Species recorded feeding on three or more plant families are regarded as polyphagous, those on two plant families as oligophagous, and as monophagous if on a single plant family.

Biological and habitat information is annotated directly to the checklist.

Broad definitions of habitats based on plant community structure are as follows:

Rainforest - tall closed forest typically dominated by Nothofagus cunninghamii and/or Atherosperma moschatum, with understoreys ranging from bryophytes/ferns to increasing complexity with shrubby Araliaceae (Pseudopanax), Epacridaceae (Cyathodes, Prionotes, Trochocarpa), Proteaceae (Cenarrhenes, Orites), Cunoniaceae (Anodopetalum, Bauera), Escalloniaceae (Anopterus), etc. Botanicaldiversity is relatively low in higher plants.

Wet eucalypt forest - tall sclerophyll forest dominated by Eucalyptus regnans, E. obliqua, E. delegatensis, E. johnstonii or E. globulus in rainfall regimes exceeding $1000 \mathrm{~mm}$ annually; understoreys may include ferns, Asteraceae (Bedfordia, Helichrysum, Olearia, Senecio), Epacridaceae (Cyathodes, 
Epacris, Richea), Proteaceae (Banksia, Lomatia, Orites, Telopea), Fabaceae (Acacia, Pultenaea), Rhamnaceae (Pomaderris) and Rutaceae (Phebalium, Zieria).

Dry eucalypt forest - open sclerophyll forest to woodland dominated by various eucalypts, especially the peppermint group (e.g. E. amygdalinaetc), sometimes with co-dominants such as Acacia and Allocasuarina; shrub and understorey species typically diverse and may prominently include Asteraceae (Cassinia, Helichrysum, Olearia), Campanulaceae (Wahlenbergia), Dilleniaceae (Hibbertia), Epacridaceae (Astroloma, Epacris, Monotoca), Fabaceae (Acacia, Dilluynia, Pultenaea), Goodeniaceae (Goodenia), Liliaceae (Dianella), Myrtaceae (Leptospermum), Pittosporaceae (Billardiera, Bursaria), Poaceae (Danthonia, Poa, Stipa, Themeda), Santalaceae (Exocarpus, Leptomeria), Sapindaceae (Dodonaea), Thymelaeaceae (Pimelea) and Xanthorrhoeaceae (Lomandra). Mixed forest - incorporates elements of rainforest and wet eucalypt forest and, if undisturbed by fire, climaxes in rainforest due to shading of eucalypt seedlings. Botanical diversity can be high, and some shrubs such as Winteraceae (Tasmannia) and Rutaceae (Eriostemon, Zieria) are typical. Coastal heath - developed on well-drained soils of the east and north coasts, including the Bass Strait islands; botanically diverse with major elements of Asteraceae (Helichrysum), Casuarinaceae (Allocasuarina), Dilleniaceae (Hibbertia), Epacridaceae (Epacris, Leucopogon, Monotoca), Fabaceae (numerous genera), Lauraceae (Cassytha), Myrtaceae (Callistemon, Kunzea, Leptospermum, Melaleuca), Polygalaceae (Comesperna), Proteaceae (Banksia, Hakea, Persoonia),
Rhamnaceae (Spyridium), and Thymeleaceae (Pimelea); much of this flora at the species level is shared with southeastern Australia.

Alpine heath - developed on alpine plateaux generally above $1000 \mathrm{~m}$ altitude; botanically diverse at the species level and in growth form, and endemism high; well represented are Asteraceae (Abrotanella, Helichrysum, Celmisia, Senecio), Apiaceae (Aciphylla), Epacridaceae (Archeria, Cyathodes, Dracophyllum, Epacris, Pentachondra, Richea), Liliaceae (Astelia, Milligania), Myrtaceae (Baeckea, Leptospermum), Onagraceae (Epilobium), Plantaginaceae (Plantago), Proteaceae (Bellendena, Grevillea, Orites), Ranunculaceae (Anemone, Ranunculus), Rosaceae (Acaena, Geum, Rubus) and Scrophulariaceae (Euphrasia, Ourisia).

Moorland heath - developed on poorly-drained peaty soils of low nutrient status mainly at low to intermediate altitudes on modest topography in the south west; major monocot groups are Cyperaceae (especially button-grass, Gymnoschoenus) and Restionaceae, while shrubby dicots include Epacridaceae (Epacris, Sprengelia), Myrtaceae (Baeckea, Callistemon, Leptospermum, Melaleuca) and Proteaceae (Banksia). Fire prone.

Abbreviations used: ANIC, Australian National Insect Collection, Canberra; TFC Tasmanian Forestry Commission, Hobart; em - emerged; i - introduced to Tasmania; ${ }^{*}$ - endemic to Tasmania.

Unless otherwise stated, all voucher specimens are housed at the Department of Primary Industry Laboratories, Hobart.

\section{ANNOTATED CHECKLIST OF TASMANIAN TORTRICIDAE}

Two subfamilies of Tortricidae occur in Tasmania.

\section{Subfamily TORTRICINAE}

Five of the nine tribes recognised by Horak (1984) are represented in Tasmania.

\section{AROTROPHORA group}

The following genera constitute a unique Australian radiation reliant where known upon Proteaceae (Common 1963). Formerly associated with the tribe Cnephasiini, they are misplaced in this tribe based upon recent morphological (Powell \& Common 1985) and biochemical (Horak et al. 1988) evidence. Their relationship to other Tortricidae remains unresolved.

\section{AROTROPHORA Meyrick, 1881}

Open forest to heathland, at elevations $0-1000 \mathrm{~m}$. Eight species known from western, southern and eastern Australia. Known larvae are internal feeders in the flower spikes of Banksia (Common 1963), but $A$. euides, a Tasmanian endemic, is on Lomatia.

1. anemarcha (Lower, 1902) (Tortrix)

Heathland and open forest. 0-200 m.

Foodplant: Proteaceae: Banksia marginata Cav., a low to tall shrub widespread in open forest and heathland from sealevel to over $1000 \mathrm{~m}$.

$3 \mathrm{~km} \mathrm{~N}$ of Eddystone Point, larva 16.ix.1985, em. 12.i.1986, P.B. McQuillan.

2. arcuatalis (Walker, 1865) (Scopula)

charistis Meyrick, 1910

submarginellus (Walker, 1866) (Crambus)

transcissella (Walker, 1866) (Eromene)

Heathland and open forest. $0-1000 \mathrm{~m}$ :

Foodplant: Proteaceae: Banksia marginata Cav.

Mt Wellington, $800 \mathrm{~m}$, larva 14.xi.1988, em. 22.iii.1989, P.B. McQuillan.
3. euides (Turner, 1927) (Tortrix)*

Subalpine open woodland. 860-950 m.

Foodplant: Proteaceae: Lomatia polymorpha R. Br., a tall shrub in subalpine open woodland.

Reservoir Lakes near Mt La Perouse 860 m, larva 16.i.1988, em. 2.ix.1988, P.B. McQuillan.

This is the first record of this endemic species since the holotype collected on Mount Wellington in 1925. Larvae tie leaves to form a large frass-filled shelter. Common (1963) was uncertain of the generic placement of this species based upon male genitalic characters and the foodplant and feeding mode cast further doubt. Females, which might clarify the situation, remain unkown.

4. siniocosma Turner, 1926 (Arotrophora). New record for Tasmania. Coastal heathland.

Foodplant: Proteaceae: Banksia marginata Cav.

Nine mile beach, larva 1.v.1983, em. 16.xii.1983, P.B. McQuillan Asbestos Range National Park, larva 27.ix.1986, em. 4.xi.1986, P.B. McQuillan.

PARAPHYAS Turner, 1927

Wet sclerophyll forest. A single species in WA and Tasmania. Foodplant unknown.

1. callixena Turner, 1927 (Paraphyas)

Wet sclerophyll forest.

\section{PERAGLYPHIS Common, 1963}

Wet and dry sclerophyll forest to heathland, at altitudes $0-1100 \mathrm{~m}$. Fourteen Australian and one Indian species. Known larvae are leaftiers on Proteaceae such as Banksia, Dryandra and Grevillea (Common 1963). One mainland species is a minor pest of silky oak, Grevillea robusta. 
1. aderces Common, 1963 (Peraglyphis)

Open forest.

2. anaptis (Meyrick, 1910) (Arotrophora)*

Coastal heath.

3. dyscheres Common, 1963 (Peraglyphis)

Open forest.

4. hemerana (Meyrick, 1882) (Arotrophora)

Montane open forest.

5. lividana (Meyrick, 1881) (Arotrophora)

Open forest.

SYLLOMATIA Common, 1963

Sclerophyll forest to heathland. Three species in eastern Australia. Known larvae monophagous on Lomatia (Common 1963).

1. pirastis (Meyrick, 1910) (Arotrophora)* castanea (Meyrick, 1910) (Arotrophora) pantoeodes (Turner, 1939) (Arotrophora)

Open forest.

Foodplant: Proteaceae: Lomatia tinctoria R. Br., a low understorey shrub in open forest.

Ridgeway, larva 16.x.1988, em. 11.ii.1989, P.B. McQuillan.

Orford, larva 26.xi.1990, em. 2.iii.1991, P.B. McQuillan.

Larvae tie terminal leaves to form a shelter densely packed with frass.

2. xythopterana (Meyrick, 1881) (Arotrophora)

Open forest. ammodes (Meyrick, 1910) (Arotrophora)

SYMPHYGAS Common, 1963*

Subalpine open forest, $950-1100 \mathrm{~m}$. One species, endemic to Tasmania. Foodplant unknown.

1. nephaula (Meyrick, 1910) (Tortrix)*

Subalpine open forest.

\section{Tribe PHRICANTHINI}

A single genus is present in Tasmania. All known larvae feed on Dilleniaceae.

\section{SCOLIOPLECTA Meyrick, 1881}

NEUROSPADES Turner, 1945

Dry sclerophyll forest to hearhland at low altitudes. Seven species Australia-wide.

1. comptana (Walker, 1863) (Sciaphila)

Coastal heath to open eucalypt forest.

Foodplant: Dilleniaceae: Hibbertia

Notes: Eggs and oviposition pattern described by Powell \& Common (1985).

2. exochus Common, 1965 (Scolioplecta)

Coastal heath.

\section{Tribe SCHOENOTENINI}

All four Tasmanian genera belong to the Proselena group of genera recognised by Common (1965). Tracholena and Syncratusare allied to the New Zealand genus Maoritenes as "archetypic Schoenotenini" (Dugdale 1966). Known larvae in this group are monophagous internal feeders.

\section{PALAEOTOMA Meyrick, 1881}

TRACHYPTILA Turner, 1916

Eucalypt forest. A monotypic genus widespread in southern Australia.
1. styphelana Meyrick, 1881 (Palaeotoma) haplopolia (Turner, 1945) (Paraselena) melanosticha (Turner, 1916) (Trachyptila) melanosticha (Turner, 1945) (Acropolitis) pellochroa (Turner, 1945) (Lamyrodes)

Wet and dry eucalypt forest.

Foodplant: Galls growing on Myrtaceae: Eucalyptusspp. (Common 1965).

Eucalyptus amygdalina Labill., leaf gall.

Blackmans Bay, em. 4.vii. 1978, R. Bashford (TFC).

Eucalyptus globulus Labill., stem galls.

Bridgewater, em. 31.iii.1981, R. Bashford (TFC).

Lake Leake, larva 13.xi.1979, em. 22.xi.1979, R. Bashford (TFC).

Eucalyptus viminalis Labill.

Rose Bay, em. 10.vii.1981, R. Bashford (TFC).

Notes: Larvae are internal feeders, hollowing out galls.

\section{PROSELENA Meyrick, 1881}

$$
\text { PARASELENA Meyrick, } 1910
$$

Open eucalypt forest. Two species in sourheastern Australia.

1. annosana Meyrick, 1881 (Proselena)

Foodplant: Pittosporaceae: Bursaria spinosa Cav, an abundant tall shrub in dry sclerophyll forest.

Mt Nelson, $200 \mathrm{~m}$, larvae in March 1986, failed to emerge but identified from Common (1965).

Young larvae are internal leaf-miners later forming a shelter from adjacent leaves spun together which are also mined.

SYNCRATUS Common, 1965

Wet eucalypt forest, mixed forest and rainforest. Two species in eastern Australia. Foodplant unknown.

1. scepanus Common, 1965 (Syncratus)

Wet eucalypt forest and mixed forest.

TRACHOLENA Common, 1965

Open forest. Three species in eastern Australia.

1. sulfurosa (Meyrick, 1910) (Cnephasia). New record for Tasmania. Open forest.

Foodplant: Native host probably Cupressaceae: Callitris spp., but recorded by Common (1965) from Cupressus in gardens.

Larvae tunnel in bark.

Tribe ARCHIPINI (s. lat.)

A large tribe with larvae tying and consuming the live foliage of a wide range of plants; a minority are shoot borers or facultative borers in fruits.

ACROPOLITIS Meyrick, 1881

Wet and dry sclerophyll forests, at altitudes $0-1200 \mathrm{~m}$. There is a strong association with live Eucalyptus and Acacia foliage but several species are polyphagous.

1. dolosana (Walker, 1863) (Tortrix)

Open forest.

2. ergophora Meyrick, 1910 (Acropolitis)

Subalpine open eucalypt forest. $860-1100 \mathrm{~m}$.

Foodplant: Cunoniaceae: Bauera rubioides Andrews.

Lyell Highway, 1976, P. Geier (I.F.B. Common, pers. comm.) Myrtaceae: Eucalyptus coccifera Hook. f.

Mt Wellington, $1100 \mathrm{~m}$, larva 26.x.1988, em. 30.xii.1988, P.B. McQuillan.

Mt Field National Park, 1080 m, pupa 11.xi.1988, em. 7.xii.1988, P.B. McQuillan.

Santalaceae: Exocarpossp.

Blackmans Bay, larva 29.x.1986, em. 17.xi.1986, R. Bashford (TFC). 
Notes: Larvac tie 2 or 3 leaves of the foodplant in December-March. Specialisation on live young eucalypt leaves by archipines is unusual; competition for this resource from other moth and beetle larvae can be intense.

Larvae in the ACT recorded on Fabaceae: Daviesia by Horak et al. 1988.

3. excelsa Meyrick, 1910 (Acropolitis)

Open forest.

4. impletana (Walker, 1863) (Tortrix)

Wet and dry eucalypt forest.

\section{5. lignigerana (Walker, 1863) (Paedisca)} inconcisana (Walker, 1863) (Sciaphila)

Wet and dry eucalypt forest.

6. ptychosema Turner, 1927* (Acropolitis)

Open forest.

Foodplant: Myrtaceae: Eucalyptus regnans F. Muell.

Briganti's Road, Florentine Valley, larva 3.iii. 1980, em. 15.iv.1980, R. Bashford (TFC).

Eucalyptus delegatensis R. Baker

M.G. Road, Cygnet River, larva 13.vii.1981, em. 25.viii.1981,

R.Bashford (TFC).

Eucalyptus amygdalina Labill.

Blackmans Bay, larva 23.xii.1981, em. 20.i.1982, R. Bashford (TFC).

Eucalyptus obliqua L'Herit.

Bicheno, larva 22.vii.1982, em. 18.viii.1982, R. Bashford (TFC).

Florentine Valley, em. 15.iv.1980, R. Bashford (TFC).

Callistemon viminalis (Gaert.) G.Don (i)

Devonport, 1976, P. Geier (ANIC).

\section{7. rudisana (Walker, 1863) (Sciaphila) indecretana (Walker, 1863) (Penthina) eucycla (Turner, 1916) (Capud)}

Virtually ubiquitous in habitats below $600 \mathrm{~m}$.

Foodplants: Fabaceae: Trifolium repens L. (i), an introduced forage plant.

Grove, larva 30.iv.1979, em. 21 .v. 1979, M.A. Williams.

Fabaceae: Medicago sativa L. (i), an introduced forage plant.

Hayes, larva 16.ii.1972, em. not dated, R.J. Hardy.

Rosaceae: Rubus ursinus Cham. \& Schlechtend. var. loganobaccus L.H. Bailey (i), an introduced horticultural plant.

Grove, larva 22.ii.1953, em. 12.iii.1953, K.A. Pickett.

Cannabaceae: Humulus lupulus L. (i), an introduced horticultural plant.

NE coast, larva no date, em. 23.ii.1971.

Larva described by Cordingley \& Danthanarayana (1976) polyphagous on a wide range of herbaceous and woody plants and achieving minor pest status on some horticultural crops.

8. xuthobapta'Turner, 1945. (Acropolitis) New record for Tasmania. Open eucalypt forest.

Foodplant: Myrtaceae; Eucalyptus camaldulensis Dehn. (i) Blackmans Bay, em. 3.i.1984, R. Bashford (TFC).

AUTHOMAEMA Turner, 1916

Eucalypt forests at low altitudes. Larval foodplants unknown.

1. diemeniana (Zeller, 1877) (Conchylis)

Open eucalypt forest.

2. pentacosma (Lower, 1900) (Anatropia)

Open eucalypt forest.

COELOPTERA Turner, 1945

Rainforest and wet sclerophyll forest, especially damp ferny gullies.
1. epiloma (Lower, 1902) (Capua). New record for Tasmania.

Notes: Adults active in xii, i.

CRYPTOPTILA Meyrick, 1881

Three described species in eastern Australia.

1. immersana (Walker, 1863) (Teras). New record for Tasmania. Rainforest margins to wet eucalypt forest.

Foodplant: Lamiaceae: Prostanthera lasianthos Labill. A tall luxuriant shrub of rainforest or wet eucalypt forest margins.

Rosebery, larva 22 xii.1983, em 13. iii.1984, P.B. McQuillan.

Mt Wellington, $550 \mathrm{~m}$, larva 10.xii.1989, em. 1.iv.1990, P.B. McQuillan..

Liliaceae: Lapageria rosed Ruiz. \& Pav. (i) (Chilean bellflower). Launceston; larva iii.1991. em. 15.iv.1991, P.B. McQuillan.

Notes: The distinctive larvae, illustrated by Common (1990), tie the terminal leaves of the spring flush, reaching the final instar in December and January. Larvae can be locally abundant, infesting at least $20 \%$ of available foliage on individual plants. This species, the so-called ivy leafroller, is known to be highly polyphagous on mainland Australia on both native and introduced hosts (Common 1990).

Turner's (1928) Tasmanian record of C. australana (Lewin, 1805), which is oligophagous on Araliaceae and Icacinaceae (Powell \& Common 1985), is almost certainly a misidentification for this species.

\section{EPIPHYAS Turner, 1927} AUSTROTORTRIX Bradley, 1956

Rainforest, wet and dry sclerophyll forest, forest-shrubland ecotones, alpine and lowland heathland, agricultural and urban habitats. At least 30 species in southern and eastern Australia. Common (1961) has clarified the limits of the genus, which is closely relared to Merophyas and Ericodesma.

\section{1. ashworthana (Newman, 1856) (Tortrix) responsana (Walker, 1863) (Teras) secundana (Walker, 1863) (Pandemis)}

Open eucalypt forest. $0-860 \mathrm{~m}$.

Foodplants: Mimosaceae: Acacia dealbata Link, a tree in open forest or at edges of tall wet forest.

Mt Nelson, 200 m, larva ix.1986, em. 3.xi.1986, P.B. McQuillan. Devonport, larva 22.ix.1986, em 15.x.1986, L. Hill.

Woodsdale, em. x. 1980, H.J. Elliott (I.F.B. Common, pers. comm.) Acacia mearnsii De Wild.

Retreat, em. x.1980, H.J. Elliott (I.F.B. Common, pers. comm.) At least two generations annually in Tasmania. Most commonly reared from bipinnate-leaved acacias, but recorded from phyllodinous A. longifolia (Andrews) Willd. in New South Wales (I.F.B. Common, pers. comm.)

2. aulacana (Meyrick, 1881) (Tortrix) asthenopis (Lower, 1902) (Tortrix)

Open forest, at altitudes $0-300 \mathrm{~m}$.

3. caryotis (Meyrick, 1910) (Tortrix)

Rainforest and wet eucalypt forest, especially at higher altitudes. Foodplants: Fagaceae: Nothofagus cunninghamii (Hook.) Oersted Mr Field National Park, $650 \mathrm{~m}$, final instar larva 26.iv.1988, ęm 4.x. 1988, P.B. McQuillan.

Myrtaceae: Eucalyptus regnans F. Muell.

Maydena, 27.i.1961, K.W. Cramer (ANIC).

Notes: This is a montane-alpine species on the southeastern mainland of Australia where it is evidently a polyphagous species, being reared from Bossiaea, Cassinia, Daviesia, Tasmannia, Epacris, Oxylobium, Parahebe, Pimelea, Pinus, and Polyscias (ANIC; I.F.B. Common, pers. comm.).

4. cerussata (Meyrick, 1910) (Tortrix)

Montane forest and wet eucalypt forest, above $400 \mathrm{~m}$. Foodplant: Asteraceae: Olearia phlogopappa (Labill.) DC. 
Mt Wellington, 400-1000 m, larvae in October 1985, em. 22.xii.1985-16.i.1986, P.B. McQuillan.

Notes: Also reared from this host, NSW, $8 \mathrm{~km}$ NNW of Jindabyne, 1000 m, 31.iii.1971, I.F.B. Common \& E.D. Edwards (ANIC).

5. cetrata (Meyrick, 1910) (Tortrix)*

Open forest.

6. dotatana (Walker, 1863) (Teras) tanyptera (Meyrick, 1910) (Tortrix)

Montane forest and wet eucalypt forest.

Possibly associated with Mimosaceae: Acacia melanoxylon R.Br. according to Meyrick (1910).

On mainland Australia reared from Fabaceae: Daviesia sp. (I.F.B. Common, pers. comm.).

7. erysibodes (Turner, 1916) (Tortrix)

Open forest.

Foodplant: Asteraceae: Olearia ramulosa [extralimital]

[A.C.T.Mt Gingera $1700 \mathrm{~m}, 17$. .ii.1950, I.F.B.Common (ANIC)].

8. eucyrta Turner, 1927 (Epiphyas)*

leuropa (Turner, 1939) (Tortrix)

Wet heaths at low altitudes. Common in habitats with Melaleuca shrubs.

9. fabricata (Meyrick, 1910) (Tortrix)

Montane forest and wet eucalypt forest.

10. flebilis (Turner, 1939) (Tortrix)* eucocephala (Turner, 1945) (Tortrix)

Wet eucalypt and mixed forest.

11. bemiphoena (Turner, 1927) (Tortrix)*

Mixed wet forest.

12. loxotoma (Turner, 1927) (Tortrix)*

Wet eucalypt forest.

13. Lycodes (Meyrick, 1910) (Tortrix)*

Open subalpine forest.

14. oresigona (Turner, 1939) (Tortrix)*

Alpine heathland. $1200 \mathrm{~m}$.

Foodplant: Asteraceae: Helichrysum ledifolium (DC.) Benth., a low aromatic shrub in alpine heathland.

Mt Wellington, $1200 \mathrm{~m}$, larvae 1.xii.1986, em 16-30.i.1987, P.B. McQuillan.

Notes: Univoltine. Similar larvae were taken from Helichrysum hookeri (Sonder) Druce near Breona on the Central Plateau but were not reared.

15. plastica (Meyrick, 1910) (Tortrix)*

Margins of wet forests at middle altitudes.

Foodplant: Asteraceae: Cassinia aculeata (Labill.) R.Br.

Woodsdale, larva 20.viii.1981, em. 11.ix.1981, R. Bashford (TFC).

16. postvittana (Walker, 1863) (Teras) basialbana (Walker, 1863) (Teras) consociana (Walker, 1863) (Pandemis)

foedana (Walker, 1863) (Dichelia) oenopa (Meyrick, 1910) (Tortrix) phaeosticha (Turner, 1939) (Tortrix) retractana (Walker, 1863) (Dichelia) reversana (Walker, 1863) (Dichelia) citulana (Walker, 1863) (Teras) secretana (Walker, 1863) (Teras) stipularis (Meyrick, 1910) (Tortrix) vicariana (Walker, 1869) (Dichelia)

Virtually ubiquitous in habitats below $750 \mathrm{~m}$. Multivoltine.
Foodplants (native):

Myrtaceae: Eucalyptus obliqua L'Herit.

Hobart (glasshouse), larvae 20.iii.1984, em. 26.iii.-17.iv.1984, R. Bashford (TFC).

Eucalyptus pauciflora Sieber ex Sprengel

Perth nursery, larva 2.x.1981, em. 26.x.1981, R. Bashford (TFC) Foodplants (introduced):

Brassicaceae: Brassica oleracea L. var. gemmifera. (i).

Moriarty, larva 17.v.1976, em. no date, P.E.L. Rapley.

Ericaceae: Vaccinium corymbosum L. (i).

Lymington, larva 11.ii.1987, em. no date, M.A. Williams.

Fabaceae: Trifolium repens L. (i).

New Town, larva no date, em. 26.viii.1957, L.W. Miller.

Geraniaceae: Pelargonium sp. (i).

Burnie, larva 24.i.1958, em. 10.ii.1958, K.A. Pickett.

Lamiaceae: Mentha $\mathrm{x}$ piperita (i).

Ouse, larva 27.xi.1978, M.A. Williams.

Rosaceac: Prunus armeniaca I. (i).

East Risdon, larva 24.i.1957, em. 5.iii.1957.

Penna, larva 7.ii. 1957, em. 13.iii. 1957.

Rosaceae: Prunus domestica L. (i).

Lindisfarne, larva 6.iii.1974, A Terauds.

Rosaceae: Malus domestica Borkh. (i).

Grove, larvae 12.ii.1965, 6.ii.1979, L.W Miller, M.A. Williams.

Rutaceae: Fortunella japonica (Thunb.). (i).

Hobart, larva 16.i.1980, em no date, M.A. Williams.

Saxifragaceae: Ribes nigrum L. (i).

New Norfolk, 21.xi.1974, R.J. Hardy.

New Town, 21.x.1984, R.J. Hardy

Plenry, larva 19.vii.1974, M.A. Williams.

Vitaceae: Vitis vinifera L. (i).

Legana, larva 12.iii.1979, em. no date, L.A. Miller.

Notes: This is the notorious light brown apple moth which has adapted to a vast range of mainly introduced foodplants. Aspects of its ecology are discussed by Geier \& Springert (1976). Danthanarayana (1975) lists 73 plant species in 27 families as foodplants Australia-wide.

\section{7. xylodes (Meyrick, 1910) (Tortrix) eurystropha (Turner, 1926) (Tortrix) paraplesia (Turner, 1914) (Tortrix)}

Virtually ubiquitous in habitats below $1100 \mathrm{~m}$. Foodplants (native):

Malvaceae: Asterotrichion discolor (Hook.) Melville.

Mt Nelson, 100 m, larva 10.xii.1986, em. 14.ii.1987, P.B. McQuillan.

Monimiaceae: Atherosperma moschatum Labill.

Mt Field National Park, larva 26.xi,1988, em 28.i.1989, P.B. McQuillan.

Arve Valley, larva 4.v.1983, em. 20.vi. 1983, R. Bashford (TFC). Myrtaceae: Eucalyptus gunnii Hook. f.

Hobart, em. x.1957 (I.F.B. Common, pers. comm.).

Eucalyptus regnans F.Muell.

Florentine Valley, larva 13.iii.1980, em. 9.i.1984, R. Bashford (TFC).

Euphorbiaceae: Beyeria viscosa (Labill.) Miq.

Mt Nelson, 100 m, larva 10.xii.1986, em. 30.i.1987, P.B. McQuillan.

Asteraceae: Olearia tasmanica (Hook. f.) W.M. Curtis.

$5 \mathrm{~km}$ SW of Mt Arrowsmith, larva 8.xii.1986. P.B. McQuillan \& M. Horak, em. 10.i.1987.

Cunoniaceae: Anodopetalum biglandulosum A. Cunn. ex Hook. f. Franklin River at Lyell Highway. Larva 6.xii.1986. P. B. McQuillan \& M. Horak, em. 17.i.1987.

Eucryphiaceae: Eucryphia lucida (Labill.) Baill.

Arve Valley, larvae 21.xii.1982, em. 7-14.ii.1983, R. Bashford (TFC).

Fagaceae: Nothofagus cunninghamii (Hook.) Oersted.

Mount Field National Park, 650 m, larva 1.xii.1982, em. 3.i.1983, P.B. McQuillan. 
Fagaceae: Nothofagus gunnii (Hook. f.) Oersted.

Lake Fenton, $1080 \mathrm{~m}$, larva 1.xii.1982, em. 7.i.1983,

P.B. McQuillan.

Phyllocladaceae: Phyllocladus aspleniffolius (Labill.) Hook. f.

Pelion Hut 860 m, larva iii.1991, P.B. McQuillan \& M. Horak.

Thymeliaceae: Pimelea nivea Labill.

Mount Nelson 200 m, larva 25.x.1986, em 4.xii.1986,

P.B. McQuillan.

Foodplants (introduced):

Caprifoliaceae: Lonicert sp. (i).

Rosny, larva 11.vii.1979, K.A. Pickett.

Fabaceae: Sophora tetraptera J.F. Mill. (i).

New Town, larva no date, em. 7.iii.1956, W.J. Newport.

Myraceae: Eucalyptus camaldulensis Dehn. (i).

Blackmans Bay, larva 12.i.1984, em. 26.ii.1984, R. Bashford

(TFC).

Rosaceae: Fragaria x anarassa Duchesne. (i).

Summerleas, larva no date, em. 5.v.1950, K.A. Pickett.

Rutaceae: Citrus x paradisi Macfady. (i).

Howrah, larva 5.xi.1974, M.A. Williams.

Pseudotsuga menziesii (Mirb.) Franco. (i).

Branxholm, larva no date, em. 29.ix. 1972, P.E.L. Rapley.

18. sp. n. $A .^{*}$ (genitalia slide T310 in ANIC)

Asteraceae: Olearia argophylla (Labill.) Benth.

Eaglehawk Neck, 22.ii.1949, J. Calaby (ANIC).

ERICODESMA Dugdale, 197

Open forest to heathland. Multivoltine. Several species in southern Australia and New Zealand.

1. concordana (Meyrick, 1881) (Tortrix)

Open forest and heathland.

Foodplant: Dilleniaceae: Hibbertia according to Meyrick (19I0).

2. indigestana (Meyrick, 1881) (Tortrix)

Heathland and open treeless habitats

Foodplants: Dilleniaceae: Hibbertia.

Larvae in spun shoots and leaves (Meyrick 1910)

Asteraceae: Arctotheca calendula (L.) Levyns (i) [Victoria].

Larvae in rolled and webbed leaves (Cordingley \& Danthanarayana 1976).

3. liquidana (Meyrick, 1881) (Cacaecia)

Open woodland and heathland.

Foodplant: Lamiaceae: Lavandula stoechas L. (i).

Golconda, larva no date, em. 5.iii.1970, A. Terauds.

Notes: Native foodplants unknown.

ISOTENES Meyrick, 1938

Rainforest margins to subalpine open mixed forest. Together with the Papuan Zacorisca Meyrick, this genus has been included in the tribe Zacoriscini Diakonoff but Common (1958) regarded the group as specialised members of the Archipini.

1. sp. n. $A^{*}$ New record for Tasmania.

Foodplant: Eucryphiaceae: Eucryphia lucida (Labill.) Baill

Scotts Peak Road, larva xii.1983, em 10.1i.1984, P.B. McQuillan. Notes: Larvae tie two leaves of the foodplant together and erode the adjacent epidermis. Adults of this undescribed species have also been collected in the vicinity of Eucryphia milliganii Hook. f. (Reservoir Lakes, near Mt La Perouse) in February. A mainland congenor, the orange fruitborer Isotenes miserana (Walker, 1863), is highly polyphagous on arboreal hosts and can be a pest of horticultural crops.

MEROPHYAS Common, 1963

Open habitats. Adults occur amongst low-growing herbaceous plants and their larvaefeed upon annual and perennial dicotyledonous herbs. In addition to the five species listed here, at least two undescribed species occur in Tasmania. Alpine species on mainland
Australia feed upon low herbaceous plants, including Celmisia, Craspedia, Brachycome and Ranunculus (ANIC records).

1. calculata (Meyrick, 1910) (Tortrix)

Open woodland.

2. divulsana (Walker, 1863) (Conchyitis) glaphyrand (Meyrick, 1881) (Tortrix) cauillata (Meyrick, 1922) (Tortrix) australiana (Strand, 1924) (Bactra) cnecochyta (Turner, 1945) (Tortrix) ischnosema (Turner, 1945) (Tortrix)

Open habitars including coastal dune swales and pastoral land. Foodplant: Onagraceae: Epilobium billardierianum Ser. ex DC Marion Bay, larva 13.xii.1986, P.B. McQuillan \& M. Horak. Fabaceae: Medicago sativa L. (i), an introduced perennial pastoral legume.

Brighton, larva 27.x.1986, em. 19.xi.1986, P.B. McQuillan. Plantaginaceae: Plantagosp. (i), an introduced weedy annual. Cambridge, larva 24.i.1985, em, 18.ii.1985, R. Bashford (TFC).

Notes: Larvae polyphagous. On mainland Auscralia, Common (1963) recorded this species, the lucerne leafroller, from Senecio, Taraxacum, Lactuca, Antirrhinum, Gypsophila, Mentha and Bassia. Cordingley \& Danthanarayana (1976) reared examples from capeweed, Arctotheca calendula (L.) Levyns in Victoria. Larvae tie terminal leaves from October to April.

3. immersana (Walker, 1863) (Paedisca) argillosana (Meyrick, 1881) (Dichelia) trygodana (Meyrick, 1881) (Tortrix)

Open habitats, including pastures.

Foodplants: Fabaceae: Trifolium repens $\mathrm{L}$. (i), an introduced pastoral legume and widespread weed [Victoria, Mornington Peninsula (ANIC)].

Trifolium sp. (i)

Sheffield, 1976, P. Geier (I.F.B. Common, pers. comm.)

Lupinus sp. (i)

Somerset, 1976, P. Geier (ANIC)

Malvaceae: Malva sp. (i)

Somerset, 1976, P. Geier (ANIC)

4. tenuifascia (Turner, 1927) (Tortrix)*

Montane open forest.

5. therina (Meyrick, 1910) (Epichorista) microstictis (Meyrick, 1910) (Epichorista)

Wet eucalypt forest to open montane forest.

Notes: On mainland Australia reared from Rosaceac: Acaena anserinifolia in the southeastern highlands (I.F.B. Common, pers. comm.).

PHRYCTORA species group

Sclerophyll forest. Larval foodplants unknown.

1. cosmopis Lower, 1894 (Dichelia)

Dry eucalypt forest.

PLACOXANTHA species group

Rainforest to wet eucalypt forest. Known larvae on ferns.

1. placoxantha (Lower, 1896) (Anisogona)

Rainforest, mixed forest and wet eucalypt forest.

Foodplant: Aspidiaceae: Polystichum proliferum (R. Br. ) Presl., an abundant low-growing fern along creek margins, seepages on forested hillsides, etc.

Mt Field National Park, larva xi.1989, em. 21.xii.1989, P.B. McQuillan.

$2 \mathrm{~km}$ SW of Renison Bell, pupa 12.xii.1987, em 18.xii.1987, P.B. McQuillan. 
Notes: Eggs and oviposition behaviour described by Powell \& Common (1985).

\section{2. effulgens Meyrick, 1910 (Capua)*}

Rainforest in western Tasmania.

SOBRIANA species group

Forested habitats.

1. alysidina Turner, 1927 (Tortrix)* $^{*}$

Tall wet eucalypt forest.

2. arcaria Meyrick, 1910 (Tortrix)

Rainforest margins, mixed forest.

Foodplant: Winteraceae: Tasmannialanceolata (Poiret) A.C. Smith. A common low to tall shrub of wet forests.

Hartz Mountains, larvae 12.xi. 1988, em 1 1.i.1989, P.B. McQuillan. Quamby Bluff, larvae common in December 1988, not reared. Notes: Larvae tie terminal leaves and flower clusters with copious silk. Locally very common and a majority of leaves can be tied on some plants.

\section{3. dyschroa Turner, 1927 (Tortrix)*}

Open alpine woodland.

Foodplant: Taxodiaceae: Athrotaxis cupressoides D. Don.

PineLake, $1200 \mathrm{~m}$, larvae 2.xii.1984, em 12.i.1985, P.B.McQuillan.

Notes: Larvae bore in tips of branchlets and pupate within.

\section{4. incompta Turner, 1927 (Tortrix)*} astathmeta Turner, 1927 (Tortrix)

Rainforest and mixed forest.

Foodplant: Fagaceae: Nothofagus cunninghamii (Hook.) Oersted. A dominant shrub to tall tree in rainforest to low alpine forest.

Mt Field National Park, larvae 2.xi.1986, em. 2-21.i.1987, P.B. McQuillan.

Hartz Mountains National Park, larvae 6.xii.1988, em. 26.i9.ii.1989, P.B. McQuillan.

Note: Larvae tie 4-8 leaves of the spring flush in November to February. Like the previous members of the "sobriana group" this species is monophagous on elements of the palaeo-austral flora. This is a plesiomorphic species with affinities to Williella in New Caledonia (Horak 1984).

5. ophiodesma Lower, 1902 (Cacaecia) $^{*}$

Subalpine open forest to montane forest.

6. sobriana Walker, 1863 (Dichelia)

Wet eucalypt forest.

STANDISHANA species group

Open sclerophyll forest to heathland. Larval foodplants unknown. 1. concolorana Meyrick, 1881 (Tortrix)

Wet and dry open heath.

\section{TECHNITIS species group}

Open forest to heathland; sea level to alpine but mostly montane; all endemic. Known larvae monophagous on shrubby Asteraceae (Olearia, Helichrysum), but one mainland species, desmotana Meyrick, 1881, on Myrtaceae: Baeckea, Leptospermum (ANIC records) and another, amoenana Walker, on spun shoots of Epacridaceae: Monotoca (Meyrick 1910) .

1. campylosticha Turner, 1939 (Tortrix)*

Montane heathland to open woodland, especially where Cyathodes grows.

Foodplant: Probably Epacridaceae.

2. hydractis Meyrick, 1910 (Tortrix)

Montane forest.

Notes: Reared from Asteraceae: Olearia spp. in the south eastern highlands of mainland Australia (I.F.B. Common, pers. comm.)
3. polyphrica Turner, 1927 (Tortrix)*

Subalpine.

4. technica Turner, 1939 (Tortrix)*

Foodplant: Asteraceae: Helichrystum thyrsoideum (DC.) Morris \& J.H. Willis.

Mt King William I, 800 m, larva 8.xii.1986, em. 2.i.1987, P.B. McQuillan \& M. Horak.

Notes: This foodplant also serves the closely related "Tortrix" oriarcha Meyrick in the subalpine areas of the Snowy Mountains (Powell \& Common 1985).

\section{5. telephanta Meyrick, 1910 (Tortrix)* \\ Subalpine open forest.}

UNASSIGNED to species group within Archipini 1. adoxodes Turner, 1939 (Tortrix)*

Rainforest and mixed forest at altitudes $200-1000 \mathrm{~m}$.

Foodplant: Fagaceac: Nothofagus cunninghamii (Hook.) Oersted

Mt Field National Park. Larvae 2.x.1985; em 14.xii.1985 P.B. McQuillan.

Hartz Mountains National Park. Larvae 16.x.1985; em. 22.xii. 1985 P.B. McQuillan.

Notes: Larvae bore into the unextended leaf buds in spring, sometimes tying adjacent shoots together.

\section{2. antilecta Turner, 1939 (Tortrix)}

3. campylosema Turner, 1945 (Tortrix)*

Notes: Only known from the type specimen from Flinders Island.

4. eucela Meyrick, 1910 (Tortrix)*

Montane forest.

5. haplopolia Turner, 1939 (Tortrix)* $^{*}$

Wet eucalypt forest.

6. humerellus Walker, 1866 (Crambus). New record for Tasmania. centurionana Meyrick, 1881 (Tortrix)

Eucalypt forest and coastal heathland.

Foodplant: Possibly Fabaceae (I.F.B. Common, pers. comm.).

Notes: Adults active in late winter.

7. Lathraea Meyrick, 1910 (Tortrix)

8. polymicta Turner, 1927 (Tortrix)* $^{*}$ Wet forest.

9. psapharana Meyrick, 1882 (Cacaecia) $^{*}$ Eucalypt forest.

10. spodota Meyrick, 1910 (Tortrix) Margins of montane eucalypt forest.

11. stigmatias Meyrick, 1910 (Tortrix)

Wet eucalypt forest.

12. tasmaniana Walker, 1863 (Conchylis) Coastal heath.

\section{Tribe EPITYMBIINI}

This tribe is largely developed in southern Australia (Common 1958), although extending to Papua. Its outstanding biological attribute is its dependence on leaflitter, especially that of Eucalyptus, as a food source (Common 1980).

About 26 formal and informal groupings of genus status are recognised (Common, unpublished) of which 16 occur in Tasmania.

ANISOGONA Meyrick, 1881

Rainforest to wet sclerophyll forest or mesic situations in drier 
forest. Six described species in eastern Australia. Larvae are unknown fromfield situations but leaf litter, including that of non-myrtaceous plants, is a probable food source.

1. mediana (Walker, 1863) (Pandemis)

acrothecta Turner, 1927 (Tortrix)

Rainforest, mixed forest, wet eucalypt forest.

Notes: Eggs and oviposition pattern described by Powell \& Common (1985).

\section{ASTHENOPTYCHA Meyrick, 1881 \\ ANATROPIA Meyrick, 1881}

Wet and dry eucalypt forest. About 14 species inhabit eastern and southern Australia. Known larvae reared from Eucalyptus leaf litter (Powell \& Common 1985).

1. conjunctana (Walker, 1863) (Sciaphila)

Wet and dry eucalypt forest.

2. epiglypta Meyrick, 1910 (Asthenoptycha)

Dry eucalypt forest.

\section{CLARANA species group}

Wet and dry sclerophyll habitats. Eight described species in southern and eastern Australia. No larvae are known from field collections.

1. clarana Meyrick, 1881 (Dichelia)

Eucalypt forests, gardens.

Notes: Eggs and oviposition pattern were described by Powell \& Common (1985).

\section{2. hyperetana Meyrick, 1881 (Dichelia) crepusculana Meyrick, 1881 (Palaeobia) diphtheroides Lower, 1902 (Dichelia)}

Dry eucalypt forests.

Notes: Eggs and oviposition pattern were described by Powell \& Common (1985).

\section{3. parastactis Meyrick, 1910 (Capua)* \\ Wet eucalypt forests.}

\section{CONSTRICTANA species group}

Wet and dry sclerophyll forests; urban gardens. About 14 species in southern and eastern Australia, including two in Tasmania.

\section{1. constrictana Walker, 1866 (Tortrix) montivagana Meyrick, 1881 (Dichelia)}

Eucalypt forests, suburban gardens.

Foodplant: Myrtaceae: Eucalyptus sp. Larvae reared on dead leaves (Powell \& Common 1985).

Notes: Eggs and oviposition pattern described by Powell \& Common (1985).

\section{2. ephedra Meyrick, 1910 (Capua $^{*}$ \\ Eucalypt forest.}

\section{DEBILIANA species group}

Dry sclerophyll forests. Three species in southeastern Australia. One member of this species group has been reared from Eucalyptus leaf litter.

1. pentazona Lower, 1901 (Capua)*

Common in open eucalypt forest, 200-1080m.

Notes: Abundant in Eucalyptus subcrenulata-E. coccifera forest near Lake Fenton $1080 \mathrm{~m}$ in December.

\section{EPITYMBIA Meyrick, 1881}

Wet and dry sclerophyll forest to subalpine heathland. Nine species in southern New Guinea, eastern and southeastern Australia (Horak
\& Common 1985). Known larvae appear to be associated with fallen leaf lituer (Powell \& Common 1985, Horak \& Common 1985).

\section{1. alaudana Meyrick, 1881 (Epitymbia) periopa (Meyrick, 1910) (Capua) cydropis (Turner, 1916) (Capua)}

Eucalypt forests.

Foodplant: Myrtaceae. Eucalyptus sp. dead leaves adhering to fallen twigs and branches (Horak \& Common 1985).

2. isoscelana (Meyrick, 1881) (Dichelia) thetis (Butler, 1882) (Conchylis)

Open forests to heathland, $0-1000 \mathrm{~m}$.

Foodplanr: Myrtaceae; Eucalyptussp.

Notes: Horak \& Common (1985) reported the larva occupying a shelter formed between two adjacent dead eucalypt leaves attached to a fallen twig. In Tasmania, this species can be found in coastal heath and heathy moorland distant from eucalypts and must feed on litter from other plants as well.

3. scotinopa (Lower, 1902) (Dichelia)

Dry eucalypt forest.

\section{EUPHONA species group}

Wet and dry sclerophyll forests. Seven described species in western, southern and eastern Australia.

1. euphona Meyrick, 1910 (Capua)

Wet and dry eucalypt forest.

Foodplant: Myrtaceae: Eucalyptus leaf litter (Powell \& Common 1985)

Notes: Eggs and oviposition characteristics described by Powell \& Common (1985).

\section{2. oxygrammana Meyrick, 1881 (Acroceuthes)} nimbosa Turner, 1927 (Capua)

Eucalypt forest.

\section{FUSCICEPSANA species group}

Wet sclerophyll forest. One member of this species group has been reared on Eucalyptus leaf litter (Powell \& Common 1985).

1. cirrhoptera Turner, 1927 (Capud $)^{*}$

Subalpine open forest.

\section{ISOCHORISTA Meyrick, 1881}

Wet and dry sclerophyll forest. About 12 species in southeastern Australia.

1. chaodes Meyrick, 1910 (Isochorista)

Tall wet eucalypt forest to open forest.

Foodplant: Myrtaceae: Eucalyptus sp.

Notes: Larvae skeletonise undersides of dead leaves (Powell \& Common 1985).

2. encotodes Meyrick, 1910 (Isochorista)

Tall wet eucalypt forest.

3. helota Meyrick, 1910 (Isochorista)

Tall wet eucalypt forest.

4. panaeolana Meyrick, 1881 (Isochorista)

Wet eucalypt forest to open dry eucalypt forest.

Foodplant: Myrtaceae: Eucalyptus sp.

Notes: Larvae join dead leaves on the ground (I.F.B. Common, pers. comm.).

LEUCOSTACTA species group

Rainforest to wet sclerophyll forest. 
1. leucostacta Meyrick, 1910 (Capua)

Larvae reared from Eucalyptusleaflitter (Powell \& Common 1985). Eggs and oviposition pattern described by Powell \& Common (1985).

This species may be a generalised archipine according to Horak (1984: 429).

\section{MERITASTIS Meyrick, 1910}

Sclerophyll forest at all alcicudes. About 15 species in eastern and southern Australia. All known larvae feeding in leaflitter. Larvae of this genus may be quice mobile in the litter and are attracted to freshly fallen leaves (Common 1980).

\section{1. laganodes (Meyrick, 1910) (Tortrix)}

Wet eucalypt forest.

2. lythrodana (Meyrick, 1881) (Cacoecia)

Wet and dry eucalypt forest.

Foodplant: Myrtaceae: Eucalyptus sp., dead leaf litrer (Powell \& Common 1985).

Notes: Eggs and oviposition characteristics described by Powell \& Common (1985).

3. polygraphana (Walker, 1863) (Tortrix)

Eucalypt forests.

4. psarades (Meyrick, 1910) (Tortrix)

Open eucalypt forest at low altitudes.

Notes: Univoltine, with adults active in mid winter.

\section{RUPICOLANA species group}

Wet and dry sclerophyll forest. About 25 species in southeastern Australia.

Known larvae reared from Eucalyptusleaf litter (Powell \& Common 1985).

1. rupicolana (Meyrick, 1881) (Dipterina)

Eucalypt forests.

2. stereodes (Meyrick, 1910) (Cnephasia)

Eucalypt forests.

SPERCHIA Walker, 1869

Forests, heathland, agricultural and urban situations; virtually ubiquitous. One species in southern and eastern Australia; probably introduced to New Zealand.

1. intractana Walker, 1869 (Sperchia) obfuscatana (Meyrick, 1881) (Capua) sordidatana (Meyrick, 1881) (Capua)

Ubiquitous at altitudes below $750 \mathrm{~m}$.

Foodplant: Myrtaceae: Eucalyptus sp. Larvae reared on leaf litter (Powell \& Common 1985).

Notes: Eggs and oviposition pattern described by Powell \& Common (1985).

\section{SUBFURCATANA species group}

Sclerophyll forests, heathland, lucerne paddocks. Two species in eastern Australia.

1. schematica Turner, 1927 (Tortrix) $^{*}$

Open forest.

2. subfurcatana Walker, 1863 (Conchylis)

Open forest, heathland, lucerne paddocks.

Notes: Larvae form a portable, usually tubular leaf-roll from which they feed and later pupate within (Powell \& Common 1985). Eucalyptus leaves were accepted in captivity (Powell \& Common 1985).

\section{TARACHOTA species group}

1. asemantica Turner, 1927 (Capua) ${ }^{*}$ poliobaphes Turner, 1927 (Capua)

Open forest.

2. dryina Meyrick, 1910 (Capua)

3. eugrapta Turner, 1927 (Capua)* thaleropis Turner, 1927 (Capua)

4. mersana Walker, 1863 (Teras) chimerinana Meyrick, 1881 (Capua) olana Walker, 1863 (Capua)

Notes: Reared from dead Eucalyptus leaves on fallen twigs in New South Wales and Victoria (I.F.B. Common, pers. comm.)

\section{VACUANA species group}

1. vacuana Walker, 1863 (Conchylis) mutatana Walker, 1863 (Grapholita)

\section{UNASSIGNED TO SPECIES GROUP}

1. camacinana Meyrick, 1882 (Proselena) ${ }^{*}$ concinnula Turner, 1927 (Tortrix)

Open eucalypt forest.

2. catarrapha Turner, 1945 (Cnephasia)* $^{*}$ Open forest.

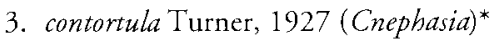
Open forest.

4. crotala Meyrick, 1910 (Cnephasia)* $^{*}$

Wet eucalypt forest.

5. euryphaea Turner, 1939 (Capua) ${ }^{*}$

Wet eucalypt forest.

6. fractifascia Turner, 1927 (Cnephasia) $^{*}$ Rainforest, mixed forest.

Foodplant: Dead leaves of Fagaceae: Nothofagus cunninghamii (Hook.) Oersted

Mt Field National Park, $800 \mathrm{~m}$. Adult emerged from litter collected 12.ix.1985 P.B. McQuillan.

7. gnophodryas Lower, 1902 (Dipterina) $^{*}$

Rainforest, mixed forest, wet eucalypt forest.

8. hyperacria Turner, 1916 (Epichorista)

9. insignana Meyrick, 1881 (Pyrgotis)

Eucalypt forest, coastal heathland.

Foodplant: Dead leaves of Eucalyptus (AN1C records).

10. leptosticha Turner, 1916 (Epichorista)

11. mermera Meyrick, 1910 (Cnephasia)

12. multistriata Turner, 1945 (Capua)* $^{*}$

13. ochrobaphes Turner, 1939 (Capua)* $^{*}$

14. phosphora Meyrick, 1910 (Cnephasia)*

Alpine heathland.

Foodplant: Dead leaves under shrubs of Asteraceae: Helichrysum ledifolium (DC.) Benth.

Mt Wellington, $1240 \mathrm{~m}$. Adult emerged from dead leaf mat sampled 30.x.1985. P.B. McQuillan. 
15. serena Meyrick, 1910 (Epichorista)* Mixed forest.

16. smenodes Meyrick, 1910 (Epichorista) $^{*}$

Open forest.

\section{UNASSIGNED TO TRIBE}

ATELODORA Meyrick, 1881

A monobasic genus. Foodplant unknown.

1. pelochytana Meyrick, 1881 (Atelodora)

Tall forest.

\section{Subfamily OLETHREUTINAE}

Larvae mostly borers or gallformers in seeds, stems and roots, some leafrollers; relatively few species are polyphagous (Powell 1980). The majority of the $3000+$ species are tropical in distribution. Of the four tribes recognised in Australia (Common 1990), two occur in Tasmania.

\section{Tribe EUCOSMINI}

Larvae of numerous Australian Eucosmini are leafrollers on myrtaceous foliage.

\section{BATHROTOMA Meyrick, 1881}

Two described species in eastern and southern Australia.

1. constrictana Meyrick, 1881 (Bathrotoma)

Open forest to coastal and moorland heath.

Foodplant: Myrtaceae: Callistemon and Melaleuca species.

Callistemon violaceus. (i)

Hobart; larvae 30.iv. 1982; em. 7-15.vi.1982. R. Bashford (TFC).

Callistemon pallidus (Bonpl.) DC.

Fern Tree; larvae 12.iv.1991. P.B. McQuillan.

Callistemon sp.

Cambridge; larvae 8.iv.1982, em. 7-23.vii.1982, R. Bashford (TFC).

Notes: Larvae live in a distinctive portable case constructed from several hollowed seed capsules stacked on each other.

\section{CROCIDOSEMA Zeller, 1847}

1. plebejana Zeller, 1847 (Crocidosema)

Gardens, vacant weedy land, roadsides.

Foodplants: Malvaceae: Lavatera, Malva, Abutilon, Hibiscus, according to Common (1990).

Notes: Larvae feed in seed capsules. A cosmopolitan species, the cotton tipworm achieves minor pest status in cotton crops.

HERMENIAS Meyrick, 1911

Sclerophyll forest. Probably associated with Eucalyptus.

1. epidola Meyrick, 1911 (Hermenias)

Eucalypt forest.

Adults active in June and July.

2. imbrifera Meyrick, 1911 (Hermenias)

Eucalypt forest.

\section{NEOHERMENIAS Diakonoff, 1966}

\section{Rainforest.}

1. sp. $A$ (aff. deloschema Turner) ${ }^{*}$

Foodplant: Cunoniaceae: Anodopetalum biglandulosum (Hook.) Hook.

Tyenna, em. 1-9.x.1990, P. Barker (TFC).

Notes: Larvae boring in seed capsules, but leaving them to pupate between tied leaves (R. Bashford, pers. comm.).
HOLOCOLA Meyrick, 1881

Open forest to alpine heathland.

1. biscissana Meyrick, 1881 (Holocola)

Coastal hearh.

2. perspectana (Walker, 1863) (Grapholita)

Open forest; heathland.

3. stilpna (Turner, 1925) (Acroclita)*

Myrtaceous heathland, $0-1100 \mathrm{~m}$.

4. thalassinana Meyrick, 1881 (Holocola)

Heathland.

Foodplant: Myrtaceae: Leptospermum, according to Meyrick (1911).

5. triangulana Meyrick, 1881 (Holocola)

Open woodland; forest margins, $0-500 \mathrm{~m}$

Foodplant: Mimosaceae: Acacia spp. (Meyrick 1881).

Galls on Acacia dealbata Link.

Black Hills, larva 2.viii.1974, M.A. Williams

Cambridge, em. 23.xi.1978, R. Bashford (TFC)

Ellendale, larva 16.vii.1974, M.A. Williams

Mount Lloyd, larva 23.vii. 1974, M.A. Williams

Notes: Larvae excavate the woody uredineine fungal galls (Uromycladium) on Acacia dealbata Link. but also eat the webbed foliage. Ecological details of this species are given by New (1982).

PALAEOBIA Meyrick, 1881

Open forest to heathland.

1. fidana Meyrick, 1881 (Palaeobia)

Wet and dry heathland.

2. longestriata Durrant, 1891 (Palaeobia)

Heathland, especially $500-1000 \mathrm{~m}$.

STREPSICRATES Meyrick, 1888

STREPSICEROS Meyrick, 1881

Eucalypt forest to subalpine heathland. A large genus in southern Australia and New Zealand with larvae amongst spun shoots of Myrtaceae.

1. argyrotypa (Turner, 1927) (Spilonota)

Subalpine heathland.

2. chalcitis (Meyrick, 1911) (Spilonota)* Open forest.

3. ejectana (Walker, 1863) (Sciaphila) igniferana (Walker, 1863) (Conchylis) saxana (Walker, 1863) (Sciaphila) servilisana (Walker, 1863) (Sciaphila)

Open eucalypt forest; coastal heath.

Foodplants: Myrtaceae: Eucalyptus amygdalina Labill., especially young foliage.

Hobart, larva 3.xii.1983, em. 9.i.1984, R. Bashford (TFC).

Notes: Also reared from Myrtaceae: Kunzea and Leptospermum on mainland Australia (Meyrick 1911). Larvae amongst webbed-up shoots and leaves.

4. euthytoma Turner, 1946 (Spilonota)*

Wet coastal heath.

5. honesta (Meyrick, 1911) (Spilonota)

Notes: Reared from Myrtaceae: Leptospermum lanigerum (Aiton) Smith in the Brindabella Range, ACT (I.F.B. Common, pers. comm.).

6. hypomolybda (Turner, 1927) (Spilonota)*

Subalpine open eucalypt forest. 
Foodplant: Myrtaceae: Eucalyptus coccifera Hook. f.

Pine Lake, Central Plateau, $1200 \mathrm{~m}$, larva 28.viii.1984, em. 23.x.1984, P.B. McQuillan.

Notes: Larva in spun-up leaves.

7. lucifera (Turner, 1946) (Spilonota)*

Alpine heath.

8. macropetana (Meyrick, 1881) (Strepsiceros)

Foodplant: Myrtaceae: Eucalyptus, according to Meyrick (1911).

Eucalyptus sp.

Hastings, em. ii.1949, J.H. Calaby (ANIC).

Notes: Larvae feeding between joined leaves.

9. morosa (Meyrick, 1911) (Spilonota)

Open forest.

10. tarachodes (Meyrick, 1911) (Spilonota)

Subalpine heathland.

11. tranquilla (Meyrick, 1911) (Spilonota)

Tall eucalypt forest.

12. zopherana (Meyrick, 1881) (Strepsiceros)

Open forest.

Notes: Reared from Myrraceae: ?Leptospermum (hedge), South Perth, 1956 (I.F.B. Common, pers. comm.).

\section{GENUS A}

\section{1. chlidana Turner, 1927 (Epiphyas)*}

Wet forest.

\section{Tribe GRAPHOLITINI}

Larvae often feeding in or on seeds.

\section{CYDIA Hubner [1825]}

1. pomonella (Linnaeus, 1758) (Phalaena) (i)

Gardens and orchards.

Foodplant: Rosaceae: Malus domestica Bork. (apple) (i)

Lindisfarne, larva 13.ii.1973, em 4.xii.1973, A. Terauds.

This is the introduced codling moth.

2. molesta (Busck, 1916) (Laspeyresia) (i)

This species, the oriental fruit moth, is regularly introduced to Tasmania in peaches grown on mainland Australia, but is not known to breed here.

\section{3. argyrocyrta Turner, 1939 (Laspeyresia) $^{*}$}

Coastal heathland.

Foodplant: Fabaceae: Glycine clandestina J. Wendl.

Rocky Cape National Park. Larvae 16.iii.1984, em. 22.x.1984

P.B. McQuillan.

Larvae live in the seed pods and consume the seeds.

\section{Excluded species}

The following species have been previously recorded for Tasmania, but should be deleted:

Alytopistis tortricitella (Walker)

A member of the family Psychidae (M. Horak, pers. comm.).

Anisogona similana Walker

A misidentification for A. mediana (Walker).

Cryptoptila australana (Lewin)

A misidentification for C. immersana (Walker).

Tortrix amoenana Walker.

Tasmanian populations have been differentiated as T. campylosticha Turner.

\section{DISCUSSION}

Relative to many other invertebrate families, Tortricidae are well represented in the Tasmanian fauna and about one third of the taxa appear to be endemic. The intermittent geographical isolation of Tasmania and its special environments and plant communities such as cool-temperate rainforest and alpine heathland gave rise to a recognisably local element in the tortricid fauna which complements the more widespread Australian element found in the eucalypt forests and coastal heathlands of eastern Tasmania.

Rainforest habitats are characteristically colonised by members of the sobriana group along with $E$. xylodes as a polyphage, some litter feeders, one or two genera of fern feeders, a single "cnephasiine" and a single eucosmine. Gapphase and forest-margin shrubs harbour monophages in the genera Isotenes and Cryptoptila.

Eucalypt forests are characterised by a high diversity of litter-feeding epitymbiines, Epiphyas and the olethreutine Strepsicrates.

Alpine heath has mostly endemic archipines such as Epiphyas and Ericodesma, while moorland heath is relatively rich in olethreutines associated with Myrtaceae.

The Tasmanian tortricid fauna is dominated by tortricines, which outnumber olethreutines sixfold. This contrasts with the situation in temperate parts of the northern hemisphere where olethreutines are dominant, but is similar to that reported for the New Zealand fauna (Dugdale 1971) and Australia generally (Common 1980) where tortricines also outnumber olethreutines. The relatively high proportion of tortricines may be partly due to the adaptation of many Australian tortricine genera to the xerophytic plant communities, especially to Eucalyptus (Common 1980). Also, unlike the Tortricinae, the large majority of Olethreutinae are derived from the Oriental fauna at a relatively recent date as reflected in their predominant presence in northern Australia (M. Horak, pers. comm.). The Australian Tortricinae are unique in respect of the Epitymbiini, mainly feeding on dead eucalypt foliage (Common 1980). However there is also evidence of considerable speciation of Archipini in alpine environments.

Tasmania is rich in primitive forms. There is indirect evidence that tortricines were pan-Gondwanic in the Cretaceous and remnants of this fauna are extant on Gondwana fragments in the southwest Pacific at least (Dugdale 1966, Horak 1984). Consistent with this hypothesis is the discovery of generalised endemic tortricines monophagous on elements of the palaeo-austral flora; 'Tortrix' incompta on Nothofagus cunninghamii, 'T.' dyschrod on Athrotaxis cupressoides and ' $T$.' arcaria on Winteraceae. Nevertheless, the Tasmanian flora has simplified considerably since the Tertiary and been invaded by xerophytic taxa as the climate became drier. These generalised tortricines are likely to be the remnants of a more diverse fauna in the past.

The morphologically generalised Arotrophora-group associated with Proteaceae appear to have no close relatives on other continents, except for an outlier in India, and possibly coevolved with this plant family. The absence of this clade of moths from the South African fauna, if confirmed, may suggest a minimum date for the appearance of this hostplant relationship after the seperation of the African landmass from Gondwana.

Several generalisations can be drawn from the patterns of foodplant utilisation as presently understood in the fauna. Asteraceae is a key family for Tasmanian tortricines, and 
appears to have been an important arena for evolution in Epipbyas especially. These plants, particularly arborescent Helichrysum and Olearia, are diverse and prominent in a variety of open or semi-open habitats at all altitudes. Ecotones between open shrubland, eucalypt forest and rainforest are rich in species and some are pioneers into newly burned habitat. Others are longer lived and important components of alpine shrubland or forest margins. Asteraceae are also significant hosts of New Zealand archipines such as Plano tortrix (Dugdale 1971). There, Olearia and its relatives support numerous species of leaf-mining Nepticulidae (Donner \& Wilkinson 1989) although this is not the case in Tasmania.

Myrtaceae feature strongly as foodplants for Tasmanian olethreutines, which consist almost exclusively of Eucosmini. Common (1980) reported that only $7 \%$ of reared Australian olethreutines were restricted to Eucalyptus foliage and none feed upon dead leaves. However, Bassian Eucosmini in general appear to be reliant on this foodplant family. This is in marked contrast to the very low incidence of tortricines on living myrtaceous tissue, surprising given the high apparency of this host family in most habitats and the cpitymbiine radiation based on myrtaceous leaf litter. Although competition by other insects for living eucalypt tissue can be intense this is unlikely to explain why so few tortricids exploit it. Rather, the biochemical defences may be too effective for Archipini to conquer, with the notable exception of Acropolitis. The few archipines found so far to develop on Eucalyptus are species with a tendency to polyphagy perhaps mediated by broad spectrum gut enzymes which effectively deal with a wide range of plants.

Ferns tend to have a limited but specialised phytophagous insect fauna and host several monophagous tortricids in Tasmania and New Zealand. However no tortricids in California or northwestern Europe use ferns.

There is a recognisable fauna of tortricines associated with the leaf litter of Nothofagus forests, as in similar forests in New Zealand (J.S. Dugdale, pers. comm.). Hence the litter-feeding habit, an austral phenomenon, is likely to predate the rise to dominance of the eucalypt forests. Whether or not the litter tortricines of the cool temperate rainforests are ancestral to the epitymbiine fauna of eucalypt forests remains unexplored.

Whereas coniferous trees are a major class of foodplants for numerous olethreutines in the northern hemisphere (Powell 1980), none are known from the Tasmanian gymnosperms (i.e. Phyllocladaceae, Podocarpaceae, Cupressaceae and Taxodiaceae). Only three Tasmanian tortricines utilise native conifers and one of these is highly polyphagous on angiosperms as well. There are also fewer conifer-feeding archipines in New Zealand than expected for the diverse gymnosperm flora (Dugdale 1977).

Other significant elements in the Tasmanian flora seem under-exploited by tortricids. No grasses are used as foodplants, in contrast to New Zealand where Poa and Cortaderia are utilised (Dugdale 1977), and Casuarinaceae, which otherwise have a rich and unusual moth fauna, support no tortricids in Tasmania. Acacia and Fabacae also seem to support few species considering their abundance in lowland habitats.

Foodplant patterns may differ on a regional basis. It is noteworthy that two taxa recently recognised in the Tasmanian fauna (Cryptoptila immersana and Isotenes sp. A.) have mainland counterparts which are exceedingly polyphagous, yet here retain a restricted host range. On the available data, a high ptoportion of the Tasmanian tortricids appear to be monophagous. This is similar to the situation in New Zealand reported by Dugdale (1977) but in contrast to the pattern observed in northwest Europe. In New Zealand the monophagy:polyphagy ratio is $47: 20$ but in Europe is 17:82. This could partly reflect more complete records for the northern hemisphere but the trend is probably reliable. Polyphagy in the Tasmanian tortricines is most marked in E. xylodes which attacks a wide range of trees and shrubs in rainforest, mixed forest and eucalypt forests. E. postvittana and $A$. rudisana have adapted to numerous introduced plants but take only a limited range of native hosts.

A high proportion of the endemic species in Tasmania occur in upland biotopes, reflecting the situation in New Zealand where over half the species and several genera are alpine. Their concealed feeding habits on or within their foodplant (e.g. in rootstocks) presumably allows them to escape the rigours of the climate (Dugdale 1971).

\section{ACKNOWLEDGEMENTS}

I am grateful to the Australian Biological Resources Survey for frnancial assistance to complete this project. Mr L. Hill, Mr T. D. Semmens and Mrs M.A. Williams helped in field collections and the rearing of larvae. Mr R. Bashford (Tasmanian Forestry Commission) generously gave access to his rearing records. The Director, Department of Parks, Wildlife and Heritage, Hobart, granted permission to sample in National Parks. Dr I.F.B. Common (Toowoomba) and Dr M. Horak (CSIRO Entomology) kindly made available the results of their taxonomic studies on Tortricinae and Olethreutinae and made valuable improvements to my manuscript.

\section{REFERENCES}

Bradley, J.D., 1956: A new genus for Tortrix postvittana (Walker) and certain other Australian and New Zealand species (Lepidoptera: Tortricidae). Bull. entomol. Res. 47: $101-105$.

Buchanan, A.M., McGeary-Brown, A. \& Orchard, A.E., 1989: A CENSUS OF THE VASCULAR PLANTS OF TASMANIA. Tasm. Herb. Occ. Publ. 2. Hobart: 82 pp.

Busck, A., 1916: In Quaintance, A.L. \& Wood, W.B. Laspeyresia molesta, an important new insect enemy of the peach. J. Agric. Res., Dep. Agric. Washington D.C. 7(8): 373-377.

Butler, A.G., 1882: On a small collection of Lepidoptera from Melbourne. Ann. Mag. Nat. Hist. (5)9: 84-103.

Common, I.F.B., 1958: The genera of the Australian Tortricidae (Lepidoptera). Proc. 10th Int. Congr. Entomol. 1: 289-295.

Common, I.F.B., 1961: The generic position of the Ausrralian light brown apple moth (Lepidoptera: Tortricidae). Proc. Linn. Soc. NSW 86: 177-182.

Common, I.F.B., 1963: A new genus for the Australian lucerne leaf roller (Lepidoptera : Tortricidae). Proc. Linn. Soc. NSW88: 298-300.

Common, I.F.B., 1963: A revision of the Australian Cnephasiini (Lepidoptera: Tortricidae: Tortricinae). Aust. J. Zool. 11: 81-152.

Common, I.F.B., 1965: A revision of the Australian Tortricini, Schoenotenini, and Chlidanotini (Lepidoptera: Tortricidae: Tortricinae). Aust. J. Zool. 13: 613-726.

Common, I.F.B., 1980: Some factors responsible for imbalances in the Australian fauna of Lepidoptera. J. Lepid. Soc. 34: 286294. 
Common, I.F.B., 1990: MOTHS OF AUSTRALIA. Melbourne University Press.

Cordingley, C.L. \& Danthanarayana, W., 1976: The lepidopterous fauna of capeweed (Arctotheca calendula) in Victoria with keys for larval identification. J. Aust. Entomol. Soc. 15: 1934.

DANTHANARAYANA, W., 1975: The bionomics, distribution and host range of the light brown apple moth, Epiphyas postvittana (Walk.) (Tortricidae). Aust. J. Zool. 23: 419-437.

Donner, H. \& Wilkinson, C., 1989: Nepticulidae (Insecta: Lepidoptera). Fauna NZ 16.

Dugdaie, J.S., 1966: A revision of New Zealand Schoenorenini and Cnephasiini (Lepidoptera: Tortricidae: Tortricinae). NZ J. Sci. 9: 731-775.

DugdaiE, J.S., 1971: Entomology of the Aucklands and other islands south of New Zealand: Lepidoptera, excluding noncrambine Pyralidae. Pac. Insects Mon. 27: 55-172.

Dugdaie, J.S., 1977: Some characreristics of phytophagous insects and their hosts in New Zealand. NZ Entomol. 6: 213-221.

DurRanT, J.D., 1891: Description of a new species of Tortricidae. Proc. Linn. Soc. NSW (II) 6: 17-18.

GeIER, P.W. \& SPRINGETT, B.P., 1976: Population characteristics of Australian leafrollers (Epiphyas spp., Lepidoptera) infesting orchards. Aust. J. Ecol. 1: 129-144.

HoraK, M., 1984: Williella - a new tortricine genus from New Caledonia indicating Gondwanan distriburion for the family (Lepidoptera: Tortricidae). Entomol. Scand. 15: 423-433.

HoraK, M. \& Brown, R.L., 1991: Taxonomy and phylogeny. in Van der Geest, L.P.S. \& Evenhuis, H.H. (Eds): TORTRICID PESTS, THEIR BIOLOGY, ENEMIES AND CONTROL. Elsevier, Amsterdam.

Horak, M. \& COMMON, I.F.B., 1985: A revision of the Australian genus Epitymbia Meyrick, with remarks on the Epitymbiini (Lepidoptera: Tortricidae). Aust. J. Zool. 33: 577-622.

Horak, M., Whittle, C.P., Bellas, T.E. \& Rumbo, E.R., 1988: Pheromone gland components of some Australian tortricids in relation to their taxonomy. J. Chem. Ecol. 14: 1163 1175 .

HUBNER, J., 1825: Verzeichniss bekannter Schmettlinge [sic]. Augsberg. Signatures 20-27: 305-431.

LEWIN, J.W., 1805: PRODROMUS ENTOMOLOGY. NATURAL HISTORY OF LEPIDOPTEROUS INSECTS OF NEW SOUTH WALES. London. 18 plates.

LINNAEUS, C., 1758: SYSTEMA NATURAE, 10. Holmiae, Laurentii Salvii: $824 \mathrm{pp}$.

Lower, O.B., 1894: New Australian Heterocera. Trans. R. Soc. S. Aust. 18: 77-113.

Lower, O.B., 1896: New Australian Lepidoptera. Trans. R. Soc. S. Aust. 20: 152-170.

LOWER, O.B., 1900: Descriptions of new Australian Lepidoptera. Proc. Linn. Soc. NSW 25: 403-423.

LOWER, O.B., 1901: Descriptions of new genera and species of Australian Lepidoptera. Trans. R. Soc. S. Aust. 25: 63-98.

LowF, O.B., 1902: Descriptions of new genera and species of Australian Lepidoptera. Trans. R. Soc. S. Aust. 26: 212-247.

Meyrick, E., 1881: Descriptions of Australian micro-Lepidoptera V-VI. Proc. Linn. Soc. NSW 6: 410-536, 629-706.

MeYrick, E., 1882: Descriptions of Australian micro-Lepidoptera. Proc. Linn. Soc. NSW7: 148-202.

Meyrick, E., 1887: Descriptions of new Lepidoptera. Proc. Linn. Soc. NSW [II] 1886: 1037-1048.
Meyrick, E., 1888: Notes on New Zealand Tortricina. Trans. Proc. NZ Inst. 20: 73-76.

Meyrick, E, 1910: Revision of Australian Tortricina. Proc. Linn. Soc. NSW 35: 139-294.

Meyrick, E., 1911: Revision of Australian Tortricina. Proc. Linn. Soc. NSW 36: 224-303.

Meyrick, E., 1922: EXOTIC MICROLEPIDOPTERA. Part 16: 481-512.

Meyrick, E., 193:. Papuan Microlepidoptera. Trans. Roy. Entomol. Soc. Lond. 87: 503-528.

NEw, T.R., 1982: Lepidoptera from Uromycladium galls on Acacia. Aust. J. Zool. 30: 357-364.

NeWMan, E., 1856: Characters of a few Australian Lepidoptera, collected by Mr Thomas R. Oxley. Trans. Entomol. Soc. Lond. (n.s.) 3(8): 281-300.

Powelt, J.A., 1980: Evolution of larval foodplant preferences in Microlepidoptera. Ann. Rev. Entomol. 25: 133-159.

POWELL, J.A. \& Common, I.F.B., 1985: Oviposition patterns and egg characteristics of Australian tortricine moths (Lepidoptera: Tortricidae). Aust. J. Zool. 33: 179-216.

STRAnd, E., 1924: Lepidoptera aus Sudwest-Australien. Deutsche Entomol. Zeitschr., 'Iris' 38: 135-148.

Turner, A.J., 1914: The Lepidoptera of Ebor Scrub, N.S.W. Proc. Linn. Soc. NSW 39: 546-564.

Turner, A.J., 1916: New Australian Lepidoptera of the family Tortricidae. Trans. Roy. Soc. S.Aust. 40: 498-536.

Turner, A.J., 1925: New Australian Lepidoptera. Trans. R. Soc. S. Aust. 49: 37-60.

Turner, A.J., 1926: Studies in Australian Lepidoptera. Trans. $R$. Soc. S. Aust. 50: 120-155.

Turner, A.J., 1927: New and little-known Tasmanian Lepidoptera. Part II. Pap. Proc. R. Soc. Tasm. 1926: 119-164.

Turner, A.J., 1928: A revision of the Lepidoptera of Tasmania. Part II. Pap. Proc. R. Soc. Tasm. 1927: 29-65.

TURNER, A.J., 1939: A second revision of the Lepidoptera of Tasmania. Pap. Proc. R. Soc. Tasm. 1938: 57-115.

Turner, A.J., 1945: Contributions to our knowledge of the Australian Tortricidae (Lepidoptera). Trans. R. Soc. S. Aust. 69: 50-72.

TURNER, A.J., 1946: Contributions to our knowledge of the Australian Tortricidae (Lepidoptera). Trans. R. Soc. S. Aust. 70: 189220.

WALKER, F., 1863: List of the specimens of lepidopterous insects in the collection of the British Museum, Part XXVII, Crambites and Tortricites. British Museum, London: 1-286.

Walker, F., 1865: List of the specimens of lepidoprerous insects in the collection of the British Museum, Part XXXIV, Supplement, Part 4. British Museum, London: 1121-1533.

WALKER, F., 1866: List of the specimens of lepidopterous insects in the collection of the British Museum, Part XXXV, Supplement, Part 5. British Museum, London: 1534-2040.

WALKER, F., 1869: Characters of undescribed Lepidoptera Heterocera. London: $112 \mathrm{pp}$.

ZeLLER, P.C., 1847: Bemerkungen uber die auf einer Reise nach Italien und Sicilien gesammelten 'Schmetterlingsarten. Isis (Leipzig) 1847: viii+561-549, xii+881-914.

ZELLER, P.C., 1877: Exotische Microlepidoptera. Horae Soc. Entomol. Ross. 13: 3-493.

(accepted 1 May 1992) 